\title{
Decentralized State Estimation of Distributed Phenomena based on Covariance Bounds
}

\author{
Felix Sawo, Frederik Beutler, and Uwe D. Hanebeck* \\ * Intelligent Sensor-Actuator-Systems Laboratory, \\ Institute of Computer Science and Engineering, \\ Universität Karlsruhe (TH), Germany; \\ e-mail: $\{$ sawo|beutler\}@ira.uka.de, uwe.hanebeck@ieee.org.
}

\begin{abstract}
This paper addresses the problem of decentralized state estimation of distributed physical phenomena observed by a sensor network. The centralized approaches are not scalable for large sensor networks, because all information has to be transmitted to a powerful central processing node requiring an extensive amount of communication bandwidth and a lot of processing power. Thus, for a decentralized reconstruction of distributed phenomena, we propose a novel methodology consisting of three steps: (a) conversion of the distributed phenomenon into a lumped-parameter system description, (b) decomposition of the resulting system in order to map the description to the actual sensor network, and (c) decomposition of the density representation leading to a decentralized estimation approach. The main problem of a decentralized approach is that due to the propagation of local information through the network, unknown correlations are caused. This fact needs to be considered during the reconstruction process in order to get correct and consistent estimation results. For that reason, we employ a robust estimator (based on Covariance Bounds) for the local reconstruction update on each sensor node. By this means, the individual sensor nodes are able to estimate the local state of the distributed phenomenon using local estimates obtained and communicated by adjacent nodes only. The information about their correlations is not stored in the sensor network.
\end{abstract}

\section{INTRODUCTION}

Recent developments and miniaturization of sensor nodes make it possible to use a wireless sensor network for monitoring natural large-area phenomena. In such scenarios, the individual sensor nodes are densely deployed inside the phenomenon and only have spatially limited acquisition capabilities. By the propagation of local information between the sensor nodes, the entire distributed phenomenon can be cooperatively reconstructed. Examples for such distributed physical systems are: temperature distribution, fluid flow, structural deflection or vibration in buildings.

The major challenge is that the individual sensor nodes in the network are able to measure the physical quantity only at discrete times and discrete locations, i.e., no information between the nodes and the time steps is available. By exploiting additional background information of the physical system in the form of a mathematical model, it is possible to get meaningful and more accurate information at any desired location and time. Thus, by means of a model-based approach the entire distributed phenomenon can be reconstructed.

In the case of a centralized reconstruction approach, as it was introduced in (Rossi et al. [2004], Sawo et al. [2006], Sawo et al. [2007]), the nodes locally collect observations and propagate them through the network to a central processing node. At the central node, this information can be fused with observations obtained by other nodes. In this case, the distributed phenomenon can be reconstructed by applying a standard estimator to the entire global state vector and storing the associated correlations between the individual sensor nodes. However, this approach for the reconstruction requires a powerful central processing node, and an extensive amount of communication bandwidth. For practical applications, a decentralized approach for the reconstruction of distributed phenomenon is more efficient, which implies that the individual sub-state vectors are manipulated separately at each reconstruction step.
For the decentralized reconstruction, the individual sensor nodes rely solely on their local observations of the phenomenon and on communicated information. The reconstruction process occurs locally on the sensor nodes without a central processing node. One possible implementation would be based on a fully connected topology, where every node transmits local information to all other nodes in the network and a centralized reconstruction is performed on each node (Mutambara [1998]). In such cases, the communication and computational load is high and no significant reduction is achieved (compared to the centralized approach). For that reason, it is more beneficial when individual nodes exploit only local estimates of their adjacent neighbors without storing any information about the correlations, as visualized in Fig. 1 (a).

Besides the aforementioned Bayesian estimation approach, there are other approaches tackling the problem of decentralized estimation in sensor networks. In (Rabbat and Nowak [2004], Blatt et al. [2007]), the problem of decentralized parameter estimation of distributed phenomenona is stated as a distributed optimization problem, which minimizes a certain cost function of interest. Although these algorithms are distributed, they do not provide a recursive characteristic and thus extensive amounts of data need to be stored. The algorithm introduced in (Lopes and Sayed [2007]) is both decentralized and recursive. However, it addresses only the estimation of stationary phenomena.

The main challenge for a fully decentralized reconstruction (in terms of a Bayesian approach) is that due to the process itself, imprecisely known correlations between the states are caused, i.e., their joint statistics are simply not available. In that case, classical filtering techniques like the Kalman filter conveniently assume uncorrelated joint densities leading automatically to unjustified improvement of estimation results. Coping with this problem, estimators based on Covariance Intersection (Castellanos et al. [1997], Julier and Uhlmann [2007], Chong and Mori [2001]) were derived, which are quite common for simultaneous localization and mapping applications (SLAM). More gen- 
eral set-based approaches for robust estimation can be derived based on Covariance Bounds (Hanebeck and Horn [2001]). These methods allow to cope with unknown correlations, both for the measurement step and the prediction step, as well as allow us to consider uncertainties in the model description. Furthermore, the systematic approach allows to find tight bounding densities even with constrained correlations (Hanebeck et al. [2001]). The issue of robustness against unknown correlations and model uncertainties is the main justification of the aforementioned methods.

The novelty of this article is to lay the foundation for an efficient and fully decentralized reconstruction of distributed phenomena. The introduced methodology basically consists of three steps, visualized in Fig. 2 (a)-(c). In a first step, the distributed phenomenon is converted into a discrete-time lumpedparameter system description (system conversion). The resulting state vector characterizing the system behavior is usually high-dimensional, especially for large-area physical phenomena. Therefore, in a second step, the lumped-parameter system is decomposed in order to map the system description to the actual sensor network (system decomposition). Based on this decomposition, the density representation for the entire sensor network is decomposed leading to a decentralized reconstruction of the distributed phenomenon (density decomposition). During the reconstruction process, the unknown correlations between the individual estimates are not neglected, but rather are considered by producing conservative and consistent estimation results. Thanks to the employed Covariance Bounds filter, all the individual sensor nodes are able to perform a local reconstruction step using local estimates of adjacent nodes only, see Fig. 1 (a). In addition, the information about their correlations is not stored in the network; saving computational and communication load.

The remainder of the paper is structured as follows. In Section 2, a rigorous problem formulation for the decentralized reconstruction of distributed phenomena is given. Section 3 then introduces the conversion and decomposition of distributed phenomena leading to a lumped and decomposed system description. Section 4 is devoted to the decomposition of the density representation in terms of decomposing the covariance matrix characterizing the estimated phenomenon. In Section 5, after a short description of the Covariance Bounds filter in a tutorial fashion, the entire framework for the decentralized reconstruction is introduced (for an example distributed phenomenon). In Section 6, the performance of the fully decentralized approach is demonstrated by means of simulations as a proof of concept.

\section{PROBLEM FORMULATION}

The main goal is to design a fully decentralized reconstruction method for distributed physical phenomena monitored by a sensor network. In order to derive an efficient reconstruction, we assume that the individual sensor nodes perform a local reconstruction step based on their own observations and estimates of neighboring nodes. By this assumption the entire sensor network and thus the reconstruction process can be conveniently regarded as a lattice dynamical system, visualized in Fig. 1 (b)

The evolution of a large number of physical systems, such as temperature distribution in a plate, heat conduction, flow in ducts and in open-channels, wave propagation, and deflection of bearings, can be described in terms of a set of partial differential equations. In this paper, we consider only onedimensional linear partial differential equations for simplicity and brevity, although similar expressions can be found for the multi-dimensional nonlinear case. In its most general form, the one-dimensional linear partial differential equation is given in implicit form by
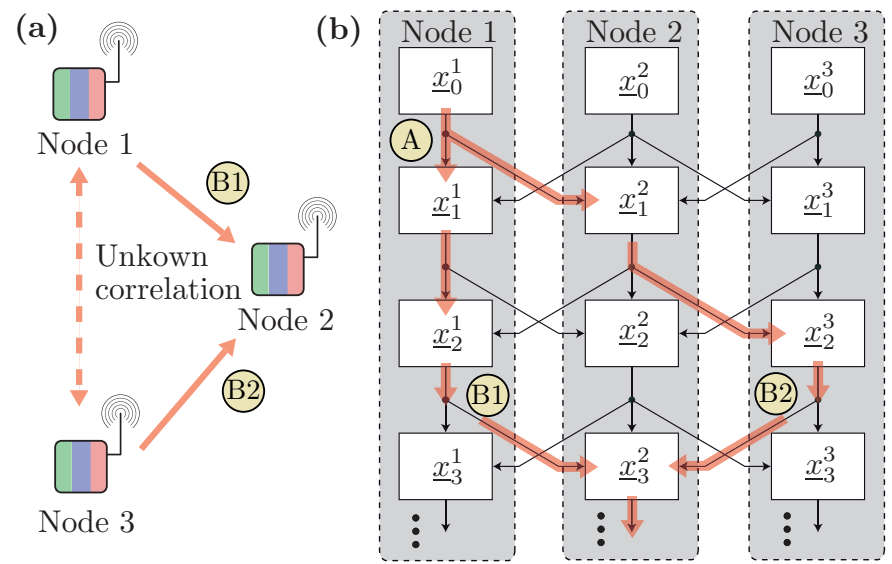

Fig. 1. (a) Each sensor locally collects observations and propagates local estimates to adjacent nodes. In a fully decentralized propagation through the network, the correlations between the individual estimates are not stored. (b) Lattice dynamical system for the visualization of the decentralized reconstruction process and cause of unknown correlations. For example, the information from Node 1 at point $\mathbf{A}$ is counted twice at point $\mathbf{B} 1$ and $\mathbf{B 2}$.

$$
\mathbb{L}\left(\boldsymbol{p}(z, t), \boldsymbol{s}(z, t), \frac{\partial \boldsymbol{p}}{\partial t}, \ldots, \frac{\partial^{i} \boldsymbol{p}}{\partial t^{i}}, \frac{\partial \boldsymbol{p}}{\partial z}, \ldots, \frac{\partial^{j} \boldsymbol{p}}{\partial z^{j}}\right)=0
$$

where $\boldsymbol{p}(z, t)$ denotes the state of the distributed phenomenon, e.g., the temperature at a certain time $t$ and certain location $z$. The source term driving the distributed phenomena is represented by $\boldsymbol{s}(z, t)$. The system input $\boldsymbol{s}(z, t)$, the state $\boldsymbol{p}(z, t)$ and its derivatives are linearly related by the operator $\mathbb{L}(\cdot)$.

Besides the conversion and decomposition of the distributed phenomenon, additional problems are the unknown correlations. In general, there are two types of sources for unknown correlations in sensor network applications. The first type is inherent to the system and caused by partially dependent noise sources for different sensor nodes. In other words, there are usually additional external disturbances affecting more than one sensor, e.g., sunshine, wind, or the same origin of a pollutant cloud. Even for ideal sensor properties, this already would lead to a partial correlation between the measured states.

On the other hand, the decentralized estimation process itself causes a second source of unknown correlations. Let us assume that a physical quantity is measured and processed only locally. In order to improve the estimate of the physical quantity, the individual sensor nodes exchange and fuse their local estimates. In this case, the resulting estimates become automatically correlated after fusing the individual estimates. Unfortunately, applying the Kalman filter to decentralized problems, while ignoring the existing dependencies between the individual states, leads to over-optimistic, even wrong estimation results. In this article, only the unknown correlations caused by the fully decentralized reconstruction itself is considered. The additional external disturbances are assumed to be uncorrelated.

\section{CONVERSION AND DECOMPOSITION OF DISTRIBUTED PHENOMENA}

In this section, we describe a method for the conversion of the partial differential equation (1) and the decomposition of the resulting system description into a decomposed system. Although the methods introduced here can be applied to general linear partial differential equations (1), we restrict our attention to a specific distributed phenomenon: the diffusion equation. 
Example 1 (Considered distributed phenomenon)

Throughout this paper, we consider the following distributed system, the one-dimensional linear partial differential equation

$$
\mathbb{L}(\boldsymbol{p}(z, t))=\frac{\partial \boldsymbol{p}(z, t)}{\partial t}-\alpha(z, t) \frac{\partial^{2} \boldsymbol{p}(z, t)}{\partial z^{2}}-\boldsymbol{s}(z, t)=0,
$$

where the diffusion coefficient $\alpha(z, t)$ could be both time and space varying. The aim is the estimation of the solution $\boldsymbol{p}(z, t)$ of the partial differential equation in a fully decentralized fashion under consideration of uncertainty influences in the system description and the measurements. That means, we assume that local state estimates are communicated only between neighboring sensor nodes and information about the resulting correlations are not stored in the network.

\subsection{Conversion of Distributed Phenomena}

The model-based state estimation of distributed phenomena based on a distributed-parameter description is quite complex. The reason is, a Bayesian estimation method usually requires the system description to be in lumped-parameter form. In order to cope with this problem, the system description has to be converted from the distributed-parameter form into a lumpedparameter form.

In general, the conversion of the distributed phenomenon (1) can be achieved by methods for solving partial differential equations, such as modal analysis (Bader et al. [2007]), the finite-difference method (Chung [2002]), the finite-element method, and the finite-spectral method (Karniadakis and Sherwin [2005]). Based on these methods, the solution domain $\Omega$ can be discretized, which results in a global state vector $\boldsymbol{x}_{k}$ characterizing the state of the distributed system. The individual entries $\boldsymbol{p}_{k}^{i}$ of the global state vector $\underline{\boldsymbol{x}}_{k}$ can be regarded as so-called software nodes, since they are only necessary for describing the distributed system in a finite state space form, see Fig. 2 (a).

The simplest method for the conversion of a distributed phenomenon is the finite-difference method. In order to solve the partial differential equation (1), the derivatives need to be approximated according to

$$
\frac{\partial \boldsymbol{p}(z, t)}{\partial t} \approx \frac{\boldsymbol{p}_{k+1}^{i}-\boldsymbol{p}_{k}^{i}}{\Delta t}, \quad \frac{\partial^{2} \boldsymbol{p}(z, t)}{\partial z^{2}} \approx \frac{\boldsymbol{p}_{k}^{i+1}-2 \boldsymbol{p}_{k}^{i}+\boldsymbol{p}_{k}^{i-1}}{\Delta h^{2}},
$$

where $\Delta t$ is the sampling time and $\Delta h$ the spatial sampling period. The superscript $i$ and the subscript $k$ in $\boldsymbol{p}_{k}^{i}$ denote the value of the distributed system at discretization node $i$ and at time step $k$. The consideration of the boundary conditions, such as Dirichlet boundary condition and Neumann boundary condition, during the conversion process is omitted in this paper; rather we refer to (Chung [2002], Rossi et al. [2004]).

\section{Example 2 (Structure of converted distributed phenomenon)}

In this example, we illustrate the structure of the converted diffusion equation (2) derived by finite differences (3). Here, we assume that the diffusion coefficient is time and space varying, and the individual coefficients $\alpha_{k}^{i}$ at location $i$ are collected in the vector $\underline{\alpha}_{k}=\left[\alpha_{k}^{1}, \ldots, \alpha_{k}^{N}\right]$. The conversion results in the following system matrix $\mathbf{A}_{k} \in \mathbb{R}^{N \times N}$,

$$
\mathbf{A}_{k}=\frac{\Delta t}{\Delta h^{2}}\left[\begin{array}{ccccc}
-2 \alpha_{k}^{1} & 1 & 0 & \ldots & 0 \\
1 & -2 \alpha_{k}^{2} & 1 & \ldots & 0 \\
\vdots & \ddots & \ddots & \ddots & \vdots \\
0 & \ldots & 1 & -2 \alpha_{k}^{N-1} & 1 \\
0 & \ldots & 0 & 1 & -2 \alpha_{k}^{N}
\end{array}\right]+\mathbf{I}
$$

where $\mathbf{I} \in \mathbb{R}^{N \times N}$ represents the identity matrix. The state of the distributed system is characterized by the state vector $\underline{\boldsymbol{x}}_{k}=\left[\boldsymbol{p}_{k}^{1}, \ldots, \boldsymbol{p}_{k}^{N}\right]$.

For the conversion of the entire distributed phenomenon, the input function $\boldsymbol{s}(z, t)$ needs to be discretized in the same way as the system state. This leads to the input vector $\underline{\boldsymbol{u}}_{k}=\left[\boldsymbol{s}_{k}^{1}, \ldots, \boldsymbol{s}_{k}^{N}\right]$. The input matrix $\mathbf{B}_{k}$ relating the input $\boldsymbol{u}_{k}$ of the distributed system to its state vector $\boldsymbol{x}_{k}$ is given by a diagonal matrix with the sampling time $\Delta t$ as the diagona entries, according to $\mathbf{B}_{k}=\operatorname{diag}\{\Delta t, \ldots, \Delta t\}$.
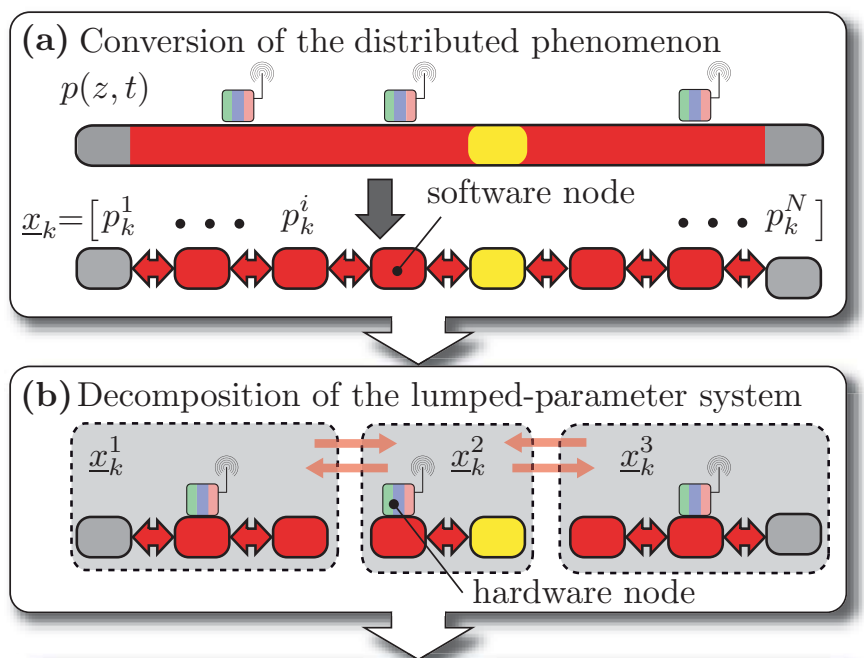

(c) Decomposition of the density representation

Fig. 2. Visualization of the three basic steps toward a fully decentralized state estimation of distributed phenomena. (a) Conversion of the distributed phenomenon into a lumped-parameter system description. (b) Decomposition of the resulting system in order to map the software nodes to respective hardware nodes. (c) Decomposition of the density representation for the decentralized estimation.

From the previous example it is obvious that in the case of the finite-difference method, the propagation of the state at a certain node $i$ can be derived by considering their respective adjacent nodes only. That means, the propagation of local estimates over time and space can be conveniently visualized by a lattice dynamical system, as shown in Fig. 1 (b). For the decentralized approach, this property is exploited so that for the reconstruction of distributed phenomena only estimates of adjacent nodes need to be considered at each update step.

In general, the conversion of linear partial differential equations (1) results in a linear system equation for the state vector $\underline{\boldsymbol{x}}_{k}$, which contains the estimates $\boldsymbol{p}_{k}^{i}$ at the individual software nodes. Adding noise terms and modelling error terms leads to the following lumped-parameter system equation

$$
\underline{\boldsymbol{x}}_{k+1}=\mathbf{A}_{k} \underline{\boldsymbol{x}}_{k}+\mathbf{B}_{k}\left(\underline{\hat{\hat{~}}}_{k}+\underline{\boldsymbol{w}}_{k}\right),
$$

where the structure of the system matrix $\mathbf{A}_{k}$ and the input matrix $\mathbf{B}_{k}$ merely depends on the applied method for converting the distributed-parameter system (1). The measurement equation providing a mapping of the finite-dimensional state vector $\underline{\boldsymbol{x}}_{k}$ to the individual measurements $\hat{y}_{k}^{i}$ at location $i$ is stated in a linear form according to,

$$
\underline{\hat{y}}_{k}=\mathbf{H}_{k} \underline{\boldsymbol{x}}_{k}+\underline{\boldsymbol{v}}_{k},
$$

where $\underline{\boldsymbol{v}}_{k}$ denotes the uncertainty in the measurements. The measurement matrix $\mathbf{H}_{k}$ is defined on the basis of geometric relations between the state vector $\boldsymbol{x}_{k}$ and the sensor locations. For a more detailed description on the derivation and the structure of the measurement matrix $\mathbf{H}_{k}$ for distributed systems, we refer to our previous research work (Sawo et al. [2006]).

\subsection{Decomposition of the Lumped-Parameter System}

For large-area distributed phenomena, the state vector $\underline{x}_{k}$ becomes high-dimensional. Due to the fact that for a centralized estimation approach the entire state vector $\underline{x}_{k}$ needs to be communicated and updated, this results in high communication costs and high computational load. For that reason, 
as a first step toward a decentralized estimation approach, a decomposition of the lumped-parameter system (5) and (6) is of major importance. Basically, the software nodes resulting from the aforementioned conversion process of the distributed phenomenon are allocated to respective hardware nodes (depending on their location). In other words, the lumped-parameter system description (5) and (6) is mapped to the sensor network, depicted in Fig. 2 (b).

For the system decomposition, the global state vector $\underline{\boldsymbol{x}}_{k}$ is decomposed into appropriate sub-state vectors $\underline{\boldsymbol{x}}_{k}^{i}$, according to

$$
\underline{\boldsymbol{x}}_{k}=\left[\underline{\boldsymbol{x}}_{k}^{1}, \ldots, \underline{\boldsymbol{x}}_{k}^{i}, \ldots, \underline{\boldsymbol{x}}_{k}^{M}\right]^{T},
$$

where each individual sub-state vector $\underline{\boldsymbol{x}}_{k}^{i}$ is allocated to appropriate hardware nodes. In a similar way, the system description (5) and (6) has to be decomposed into appropriate sub-systems. Here, we assume that the decomposed system and measurement model for the $i$-th sub-state vector $\underline{\boldsymbol{x}}_{k}^{i}$ and the $i$-th measurement vector $\underline{\hat{y}}_{k}^{i}$ is stated as follows,

$\underline{\boldsymbol{x}}_{k+1}^{i}=\sum_{j=1}^{M} \mathbf{A}_{k}^{i j} \underline{\boldsymbol{x}}_{k}^{j}+\sum_{j=1}^{M} \mathbf{B}_{k}^{i j}\left(\underline{\hat{u}}_{k}^{j}+\underline{\boldsymbol{w}}_{k}^{j}\right), \underline{\hat{y}}_{k}^{i}=\sum_{j=1}^{M} \mathbf{H}_{k}^{i j} \underline{\boldsymbol{x}}_{k}^{j}+\underline{\boldsymbol{v}}_{k}^{i}$

where $\mathbf{A}_{k}^{i j}, \mathbf{B}_{k}^{i j}$ and $\mathbf{H}_{k}^{i j}$ respresents the respective sub-matrices of the global matrices $\mathbf{A}_{k}, \mathbf{B}_{k}$ and $\mathbf{H}_{k}$.

It is important to emphasize that generally, the decomposition of the lumped-parameter system (5) and (6) into the decomposed system (7) is achieved in an exact fashion, i.e., no approximation is necessary. However, for certain applications it might be more beneficial to ignore neglectable sub-systems in (7), for instance when certain sub-matrices $\mathbf{A}_{k}^{i j}$ are close to zero. This certainly could lead to a more efficient estimation process.

Furthermore, in the deterministic case, i.e., the uncertainties in the models and in the measurements are not considered, the decomposed system description (7) can be directly used for a decentralized calculation. That means, the propagation of the distributed phenomenon can be exactly derived in a decentralized fashion. In the stochastic case, a decomposition of the density representation is necessary in order to arrive at a fully decentralized approach, introduced in the next section.

\section{DECOMPOSITION OF DENSITY REPRESENTATIONS}

This section is devoted to the decomposition of the density representation, see Fig. 2 (c). In order to derive a fully decentralized estimation approach, the decomposed density representation has to match the decomposed system equations (7). The decomposition of the covariance matrix $\widetilde{\mathbf{C}}_{k}$ of the estimated state of the distributed phenomenon is shown in Fig. 3.

In the centralized state estimation approach, all the information about the distributed system and the measurements has to be collected and processed at a central processing node. In the case of a fully decentralized estimation, the individual substate vectors $\underline{x}_{k}^{i}$ are manipulated separately at each update step, rather than the entire global state vector $\underline{\boldsymbol{x}}_{k}$. By this means, it is possible to perform the state estimation locally on each hardware node. Furthermore, only local estimation results need to be communicated between neighboring nodes, which dramatically reduces the communication load.

In a fully decentralized approach for the reconstruction of distributed phenomena, it is beneficial not to store the occuring correlations between the individual estimates. That means the cross-covariances $\mathbf{C}_{k}^{i j}$ between the sub-state vector $\underline{\boldsymbol{x}}_{k}^{i}$ and $\underline{\boldsymbol{x}}_{k}^{j}$ with $i \neq j$ are not stored in the network. Hence, the covariance matrix of the state vector $\underline{\boldsymbol{x}}_{k}$ is characterized by

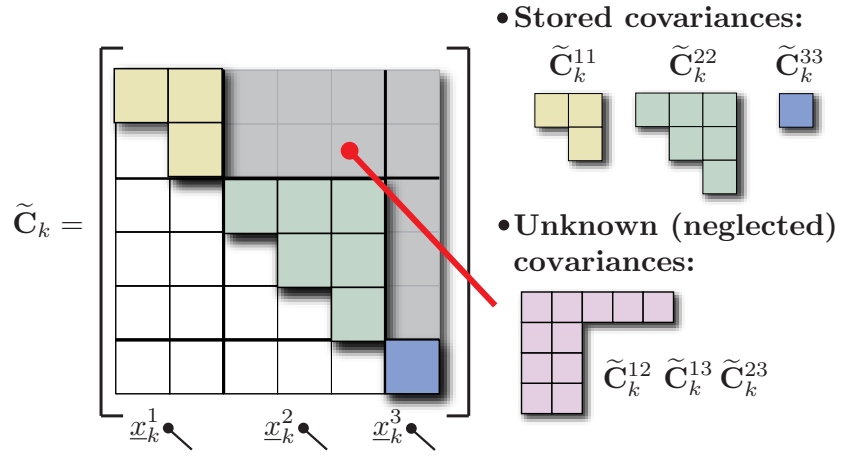

derived from system decomposition

Fig. 3. Visualization of the decomposition of the density representation. The covariance matrix $\widetilde{\mathbf{C}}_{k}$ is decomposed with respect to the achieved system decomposition. The covariances $\widetilde{\mathbf{C}}_{k}^{i i}$ are stored in the network and the crosscovariances $\widetilde{\mathbf{C}}_{k}^{i j}$ with $i \neq j$ are neglected.

$$
\widetilde{\mathbf{C}}_{k}=\operatorname{Cov}\left\{\underline{\boldsymbol{x}}_{k}^{i}, \underline{\boldsymbol{x}}_{k}^{j}\right\}=\left\{\begin{array}{ll}
\widetilde{\mathbf{C}}_{k}^{i j} & \text { for } i=j \\
\text { unknown } & \text { for } i \neq j
\end{array},\right.
$$

which reduces the computational burden, memory resources, and communication activities between the individual nodes to a minimum. The structure of the covariance matrix $\widetilde{\mathbf{C}}_{k}$ of the entire network is depicted in Fig. 3.

In order to visualize the effect of neglecting unknown correlations during the decentralized reconstruction of a distributed phenomenon, we consider the following example.

\section{Example 3 (Comparison of different reconstruction approaches)} In this example, the distributed system (2) is converted into state-space form consisting of 3 nodes. The boundary conditions are assumed to be $g_{N}=0$ at both ends. Applying the finite differences (3) and considering the boundary conditions leads to a system matrix $\mathbf{A}_{k}$ with a similar structure to (4). The initial state vector is assumed to be $\underline{\hat{x}}_{0}=[0,0,0]^{T}$ and the true state to be $\underline{\widetilde{x}}=[10,10,10]^{T}$. The estimated state vector $\underline{\boldsymbol{x}}_{k}^{e}$ can be propagated through the system equation to the next time step by means of the Kalman prediction step,

$$
\underline{\hat{x}}_{k+1}^{p}=\mathbf{A}_{k} \underline{\hat{x}}_{k}^{e}, \quad \mathbf{C}_{k+1}^{p}=\mathbf{A}_{k} \mathbf{C}_{k}^{e} \mathbf{A}_{k}^{T},
$$

where $\underline{\hat{x}}_{k+1}^{p}$ and $\mathbf{C}_{k+1}^{p}$ denote the predicted mean and covariance matrix, respectively. Furthermore, we assume that at time steps $k=$ $[10,20,30,40,50]$ a Kalman filter step is performed.

The simulation results are shown in Fig. 4 . In the case of a centralized estimation approach, the entire covariance matrix $\mathbf{C}_{k}^{e}$ is stored and considered in the update step. Hence, the estimation result can be regarded as a reference solution, as shown in Fig. 4 (a). In the decentralized approach, the cross-covariances $\mathbf{C}_{k}^{i j}$ with $i \neq j$ are not stored and thus cannot be considered in the prediction step. Simply assuming the individual states $\boldsymbol{x}_{k}^{i}$ to be uncorrelated leads to overoptimistic results, as depicted in Fig. 4 (b). It is obvious that due to the unjustified improvement of the variances, the filter step at $k=10$ has almost no influence. In comparison, robust estimators based on Covariance Bounds systematically consider the unknown correlations between the individual estimates $\boldsymbol{x}_{k}^{i}$ and thus provide a consistent and conservative estimation result, see Fig. 4 (c).

\section{DECENTRALIZED ESTIMATION OF DISTRIBUTED PHENOMENA BASED ON COVARIANCE BOUNDS}

In this section, we derive a fully decentralized estimation approach for the reconstruction of distributed phenomena characterized by partial differential equations (1). As it was shown in the previous section, neglecting the unknown correlations during the reconstruction process leads to over-optimistic and 
wrong estimation results. In order to get consistent and correct results, robust estimators are necessary, which consider unknown correlations. Here, the Covariance Bounds filter is employed for the decentralized reconstruction of distributed phenomena. By this means, the individual sensor nodes in the network locally observe the physical phenomenon and propagate their local estimates to adjacent nodes (without storing information about their correlations).

\subsection{Covariance Bounds Filter}

This section is devoted to the description of the Covariance Bounds filter in a tutorial fashion. More details about this type of bounding density and its application can be found in our previous research work (Hanebeck [2001], Hanebeck et al [2001], Hanebeck and Horn [2001]).

Let's assume that we are given $N$ individual random vectors $\underline{\boldsymbol{x}}_{k}^{i}$ with expected values and individual covariances

$$
\mathrm{E}\left\{\underline{\boldsymbol{x}}_{k}^{i}\right\}=\underline{\hat{x}}_{k}^{i}, \quad \operatorname{Cov}\left\{\underline{\boldsymbol{x}}_{k}^{i}\right\}=\widetilde{\mathbf{C}}_{k}^{i i},
$$

where the estimates $\underline{\boldsymbol{x}}_{k}^{i}$ are correlated with completely unknown correlations. In the case of distributed systems, the individual state vectors $\underline{x}^{i}$ contain the local estimates of the phenomenon to be reconstructed, e.g., local temperature or humidity values. The main goal is to find a family of bounding covariances $\mathbf{C}_{k}$ with

$$
\mathbf{C}_{k} \geq \widetilde{\mathbf{C}}_{k}
$$

for all possible joint covariances $\widetilde{\mathbf{C}}_{k}$. In our previous work (Hanebeck [2001]), we have proven that given a positive definite symmetric matrix $\widetilde{\mathbf{C}}_{k}$ with completely unknown correlations (8), a "larger" matrix can be parameterized by

$$
\mathbf{C}_{k}\left(\underline{\kappa}_{k}\right)=\operatorname{diag}\left(\frac{1}{\kappa_{k}^{1}} \widetilde{\mathbf{C}}_{k}^{11}, \quad \ldots \quad, \frac{1}{\kappa_{k}^{M}} \widetilde{\mathbf{C}}_{k}^{M M}\right),
$$

where the $\kappa_{k}^{i}$ must satisfy $0<\kappa_{k}^{i}<1$ and $\sum_{i=1}^{M} \kappa_{k}^{i}=1$. The individual parameters $\kappa_{k}^{i}$ are collected in the vector $\underline{\kappa}_{k}$. A similar parameterization of the bounding density can be found in the case of constrained correlations, i.e., symmetric constraints (Hanebeck et al. [2001]) or asymmetric constraints.

Applying the Covariance Bounds (9) for bounding the unknown covariance matrix during the estimation process results in a family of covariance matrices $\mathbf{C}_{k}^{e}\left(\underline{\kappa}_{k}\right)$ for the estimated state $\underline{x}_{k}^{e}$. The individual members of the covariance matrix $\mathbf{C}_{k}^{e}\left(\underline{\kappa}_{k}\right)$ are an upper bound for the union of all possible matrices with arbitrary correlations. Here, it is emphasized that the intersection is a tight bound for this union.

In general, it would be best to keep the entire family of bounding densities during their further processing. However, the problem is that the dimension of the vector parameterizing the member of bounding densities is increasing with every step. Hence, for practical reasons it is necessary to select a specific value $\underline{\kappa}^{*}$ after certain processing steps. The $\underline{\kappa}_{k}$ may be selected in some optimum way, for example minimizing the determinant or trace of $\mathbf{C}_{k}^{e}\left(\underline{\kappa}_{k}\right)$.

At this point, it is important to note that the systematic approach of Covariance Bounds allows to find tight bounding densities for constrained correlations (Hanebeck et al. [2001]). In addition, the application of the bounding density is not restricted to the consideration of unknown correlations, but can be extended to unknown system matrices and measurement matrices in a fairly straightforward manner.

\subsection{Decentralized Estimation of Distributed Phenomena}

As it was demonstrated in Example 3, in the case of unknown correlations, the decentralized reconstruction neglecting these

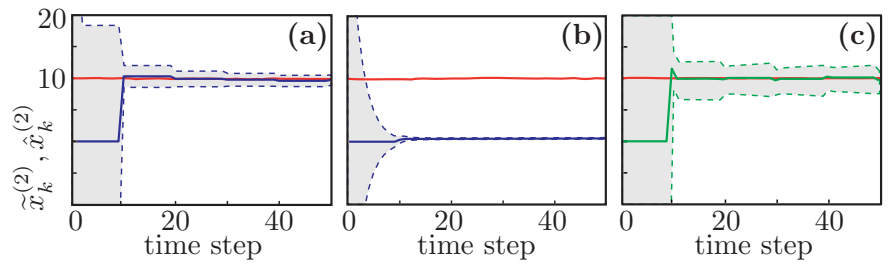

Fig. 4. Visualization of the effect of neglecting unknown correlations during the propagation of information through the network: (a) centralized Kalman filter, (b) decentralized Kalman filter with neglected correlations, (c) Covariance Bounds filter.

correlations cannot be applied since it leads to over-optimistic and even wrong results. Therefore, we derive a robust estimator based on Covariance Bounds for the decentralized reconstruction of distributed systems.

The methods introduced here can be applied to the general decomposed system (7). However, we restrict our attention to a specific structure where the individual estimates $\underline{x}_{k}^{i}$ depend only on estimates of adjacent nodes. This system structure naturally results by the application of finite-difference methods to the distributed system (2).

\section{Example 4 (Considered system structure in decomposed form)}

By applying the finite-difference method for the discretization step, the global system equations (5) and (6) can be decomposed in such a way that the local state vectors $\boldsymbol{x}_{k}^{i}$ depend only on the previous state of adjacent nodes $i-1$ and $i+1$ (see Example 2). Furthermore, it can be assumed that the global measurement matrix $\mathbf{H}_{k}$ consists only of diagonal entries. Thus, the structure of the general decomposed system (7) reduces to the following

$$
\underline{\boldsymbol{x}}_{k+1}^{i}=\sum_{j=i-1}^{i+1} \mathbf{A}_{k}^{i j} \underline{\boldsymbol{x}}_{k}^{j}+\mathbf{B}_{k}^{i i}\left(\underline{\hat{x}}_{k}^{i}+\underline{\boldsymbol{w}}_{k}^{i}\right), \quad \underline{\hat{y}}_{k}^{i}=\mathbf{H}_{k}^{i i} \underline{\boldsymbol{x}}_{k}^{i}+\underline{\boldsymbol{v}}_{k}^{i},
$$

where $i$ is the node to be considered, $i-1$ is the left neighboring node, and $i+1$ is the right neighboring node.

In general, there exist both spatial and temporal correlations inherent to the physical phenomenon to be observed. These correlations are usually caused by partially stochastic dependent and distributed noise sources affecting more than one location in the distributed system, and thus more than one sensor node. However, in this article we assume that the individual components of both the global system uncertainty $\underline{\boldsymbol{w}}_{k}$ and the global measurement uncertainty $\underline{\boldsymbol{v}}_{k}$ are uncorrelated. That means, the covariance matrices of the noise terms are characterized by

$$
\mathbf{C}_{k}^{w}=\left\{\begin{array}{ll}
\mathbf{C}_{k}^{w i j} & \text { for } i=j \\
\mathbf{0} & \text { for } i \neq j
\end{array}, \quad \mathbf{C}_{k}^{v}=\left\{\begin{array}{ll}
\mathbf{C}_{k}^{v i j} & \text { for } i=j \\
\mathbf{0} & \text { for } i \neq j
\end{array},\right.\right.
$$

which means no common source term is affecting more than one location and one sensor node. In the case of temporal and spatial correlations inherent to the distributed system it is necessary to derive an appropriate model. Thus, in this article, only the unknown correlations caused by the decentralized reconstruction process itself are considered, see (8).

Local Prediction Step The purpose of the local prediction step is to propagate the state estimate $\underline{\boldsymbol{x}}_{k-1}^{e i}$ through the system equation (10) to the next time step $k$. The prediction step can be performed based on the state estimates received from adjacent nodes. When performing a local prediction step, the mean value $\underline{\hat{x}}_{k}^{p i}$ of the predicted state $\underline{\boldsymbol{x}}_{k}^{p i}$ is simply given by the weighted sum of the mean of the previous states and the input state according to

$$
\underline{\hat{x}}_{k}^{p i}=\sum_{j=i-1}^{i+1} \mathbf{A}_{k-1}^{i j} \underline{\hat{x}}_{k-1}^{e j}+\mathbf{B}^{i i} \underline{\hat{u}}_{k-1}^{i},
$$


which obviously does not depend on the correlation between the individual local state estimates.

The problem is now to calculate the covariance matrix $\mathbf{C}_{k}^{p i i}$ of the predicted state $\underline{\boldsymbol{x}}_{k}^{p i}$ when the correlation between the individual previous states is unknown. Replacing the unknown covariance matrix $\widetilde{\mathbf{C}}_{k}^{e i i}$ in the standard Kalman prediction step by means of the Covariance Bounds (9) leads to the following equation for the prediction step

$\mathbf{C}_{k}^{p i i}\left(\underline{\kappa}_{k}\right)=\sum_{j=i-1}^{i+1} \frac{1}{\kappa_{k}^{j}} \mathbf{A}_{k-1}^{i j} \mathbf{C}_{k-1}^{e j j}\left(\mathbf{A}_{k-1}^{i j}\right)^{T}+\mathbf{B}_{k-1}^{i i} \mathbf{C}_{k-1}^{w i i}\left(\mathbf{B}_{k-1}^{i i}\right)^{T}$. It is emphasized that the resulting predicted covariance matrix $\mathbf{C}_{k}^{p i i}\left(\underline{\kappa}_{k}\right)$ depends on the parameter vector $\underline{\kappa}_{k}$.

Local Measurement Step For the purpose of reducing the estimation uncertainty, measurements are incorporated that are related to the state via the local measurement equation (10). Since we assume that there is no spatial correlation in the considered domain, the local measurement step can be performed based only on local measurements $\hat{y}_{k}^{(i)}$, i.e., exchange of measurements between the nodes is not necessary in that case. Then the mean and the covariance matrix are given by

$$
\begin{aligned}
\underline{\hat{x}}_{k}^{e i}\left(\underline{\kappa}_{k}\right) & =\underline{\hat{x}}_{k}^{p i}+\mathbf{K}_{k}\left(\underline{\kappa}_{k}\right)\left(\underline{\hat{y}}_{k}^{i}-\mathbf{H}_{k}^{i i} \underline{\hat{x}}_{k}^{p i}\right), \\
\mathbf{C}_{k}^{e i i}\left(\underline{\kappa}_{k}\right) & =\mathbf{C}_{k}^{p i i}\left(\underline{\kappa}_{k}\right)-\mathbf{K}_{k}\left(\underline{\kappa}_{k}\right) \mathbf{H}_{k}^{i i} \mathbf{C}_{k}^{p i i}\left(\underline{\kappa}_{k}\right),
\end{aligned}
$$

where the Kalman gain $\mathbf{K}_{k}\left(\underline{\kappa}_{k}\right)$ is given by

$\mathbf{K}_{k}\left(\underline{\kappa}_{k}\right)=\mathbf{C}_{k}^{p i i}\left(\underline{\kappa}_{k}\right)\left(\mathbf{H}_{k}^{i i}\right)^{T}\left(\mathbf{C}_{k}^{v i i}+\mathbf{H}_{k}^{i i} \mathbf{C}_{k}^{p i i}\left(\underline{\kappa}_{k}\right)\left(\mathbf{H}_{k}^{i i}\right)^{T}\right)^{-1}$ which depends on the $\underline{\kappa}_{k}$. Due to unknown correlations at the local prediction step and the resulting family of predicted covariances, the result of the local measurement step is a family of bounding densities parameterized by $\underline{\kappa}_{k}$.

For the next prediction and measurement step it is necessary to select an optimal $\kappa^{*}$. There are several possibilities for the selection of a specific $\underline{\kappa}^{*}$, which result in a single predicted density characterized by mean $\underline{\hat{x}}_{k}^{p i}$ and covariance $\mathbf{C}_{k}^{p i i}$. In this article, the optimal $\underline{\kappa}^{*}$ is selected immediately after the prediction step when there are no measurements $\underline{y}_{k}^{i}$ available. In the case of local measurements $\underline{\hat{y}}_{k}^{i}$, the optimal $\underline{\kappa}^{*}$ is selected after the measurement step.

\section{SIMULATION RESULTS}

In this section, the performance of the decentralized reconstruction of a distributed phenomenon based on the Covariance Bounds filter is demonstrated by some simulation results as a proof of concept. We consider the following simulation setup:

\section{Example 5 (Simulated system)}

In this simulation, we consider the one-dimensional diffusion equation (2) subject to boundary conditions corresponding to insulation at both ends and characterized by the diffusion coefficient $\alpha(z, t)=1$. The distributed phenomenon is discretized by 50 nodes with a spatial sampling period $\Delta z=0.5$ and a sampling time $\Delta t=0.05$. There exists a noisy input in the center of the considered solution domain given by

$$
\hat{u}_{k}^{(25)}= \begin{cases}100 & \text { for } 0 \leq t_{k}<200 \\ 0 & \text { for } t_{k} \geq 200\end{cases}
$$

The system noise term for the individual discretization nodes is assumed to be $C_{k}^{w i i}=0.5$. The individual initial states for the estimator are $\hat{x}_{0}^{i}=20$, whereas the true initial realization is assumed to be $\widetilde{x}_{0}^{i}=19$. Furthermore, there is a sensor node at each discretization node $p_{k}^{i}$ with a measurement noise variance $C_{k}^{v i i}=3$. At every time step, randomly chosen sensor nodes are performing a measurement step, in order to reconstruct the distributed phenomenon in a decentralized fashion.
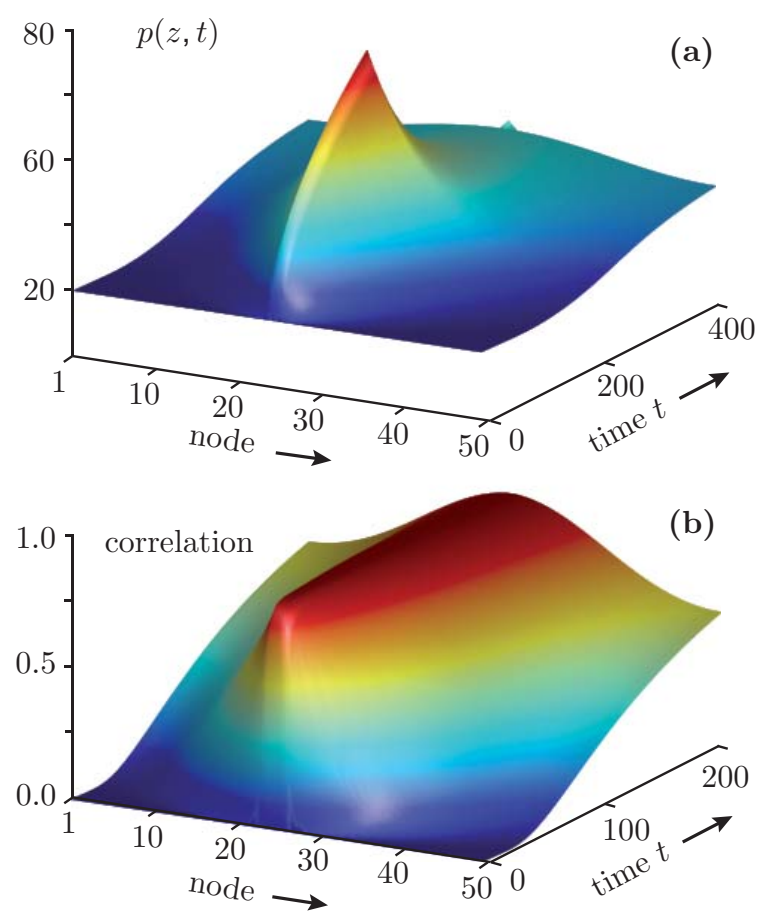

Fig. 5. (a) Numeric solution of the considered distributed phenomenon. (b) Correlation coefficient $r_{k}^{i j}$ between the individual variances of node 25 with right and left neighboring nodes. It is obvious that the entire network becomes almost fully correlated during the reconstruction (existing correlations cannot be neglected).

The numeric solution of the considered distributed phenomenon $\boldsymbol{p}(z, t)$ is depicted in Fig. 5 (a). Due to the propagation of state information, the entire network becomes almost fully correlated during the reconstruction process. In Fig. 5 (b), the correlation coefficient $r_{k}^{i j}$ between the state estimate $\boldsymbol{x}_{k}^{i}$ at discretization node 25 and the neighboring nodes is depicted. Hence, this correlation cannot be neglected during the reconstruction process.

Fig. 6 (a) shows the root mean square error (rms) for 100 Monte Carlo simulation runs averaged over all discretization nodes. At every update step, ten randomly chosen sensor nodes are performing a measurement update. The centralized Kalman filter (blue line) stores and considers the entire covariance matrix at each update step. That means, the estimation result can be regarded as the reference solution.

In the case of a fully decentralized reconstruction, the correlations between the individual estimates are not stored and thus are unknown at each update step. It is obvious from Fig. 6 (a) (red line) that neglecting the unknown correlations between the individual estimates causes over-optimistic and wrong results. This leads to the unjustified improvement of the estimates of the individual nodes. Therefore, the measurements have a minor influence on the estimation and estimation error remains. An higher number of sensor nodes or more accurate measurements would be necessary to solve this problem, as can be seen in Fig. 6 (b). The Covariance Bounds filter (green line) provides a systematic way to consider the unknown correlations between the individual estimates during the estimation process. By this means it is possible to derive conservative and consistent estimation results. 


\section{CONCLUSIONS}

This paper introduces a methodology for the decentralized reconstruction of distributed phenomena by means of a sensor network. In order to estimate the distributed state in a decentralized fashion, the system description is converted and decomposed, resulting in a network consisting of software nodes mapped to hardware nodes. For the efficient reconstruction of large-area distributed systems, local information has to be propagated through the sensor network in a fully decentralized fashion. This automatically causes unknown correlations between the individual estimates.

Besides the introduction of a novel methodology for the decentralized reconstruction of distributed phenomena, the novelty of this article is the systematic consideration of unknown correlations (caused by a fully decentralized approach). The method of Covariance Bounds is applied to derive robust estimators for both the local prediction step and the local measurement step. By this means, it is possible that individual sensor nodes locally reconstruct large-area physical systems and exchange their estimates only with adjacent nodes (without storing information about correlations). It is important to emphasize that the method of Covariance Bounds can be applied independently of the conversion and decomposition method. Furthermore, within this framework, the application of other estimators robust against unknown correlations is possible.

For the considered one-dimensional partial differential equation, the decentralized solution may seem unnecessarily involved. However, the same principles can easily be applied to multiple dimensions. The consideration of spatial and temporal correlations caused by additional external disturbances affecting more than one sensor node is left for future research work. However, it is believed that in such cases it becomes even more important to consider unknown correlations during the reconstruction steps.

\section{REFERENCES}

Thomas Bader, Alexander Wiedemann, Kathrin Roberts, and Uwe D. Hanebeck. Model-based Motion Estimation of Elastic Surfaces for Minimally Invasive Cardiac Surgery. In Proceedings of the 2007 IEEE International Conference on Robotics and Automation (ICRA 2007), pages 2261-2266, Rome, Italy, April 2007.

D. Blatt, A. O. Hero, and H. Guachman. A Convergent Incremental Gradient Method with Constant Stepsize. SIAM Journal of Optimization, 18, 2007.

J.A. Castellanos, J.D. Tardos, and G. Schmidt. Building a Global Map of the Environment of a Mobile Robot: The Importance of Correlations. In IEEE International Conference on Robotics and Automation (ICRA 1997), Albuquerque, New Mexico, 1997.

C.Y. Chong and S. Mori. Convex Combination and Covariance Intersection Algorithms in Distributed Fusion. In The 4th International Conference on Information Fusion (Fusion 2001), Montreal, Canada, 2001.

T.J. Chung. Computational Fluid Dynamics. Cambridge University Press, 2002.

Uwe D. Hanebeck. A Square-Root Algorithm For Set Theoretic State Estimation. In Proceedings of the European Control Conference (ECC 2001), Porto, Germany, 2001.

Uwe D. Hanebeck and J. Horn. An Efficient Method for Simultaneous Map Building and Localization. In Proceedings of SPIE, Orlando, Florida, 2001.

Uwe D. Hanebeck, K. Briechle, and J. Horn. A Tight Bound for the Joint Covariance of Two Random Vectors with Unknown but Constrained Cross-Correlation. In IEEE Conference on Multisensor Fusion and Integration for Intelligent Systems (MFI 2001), Baden-Baden, Germany, 2001.
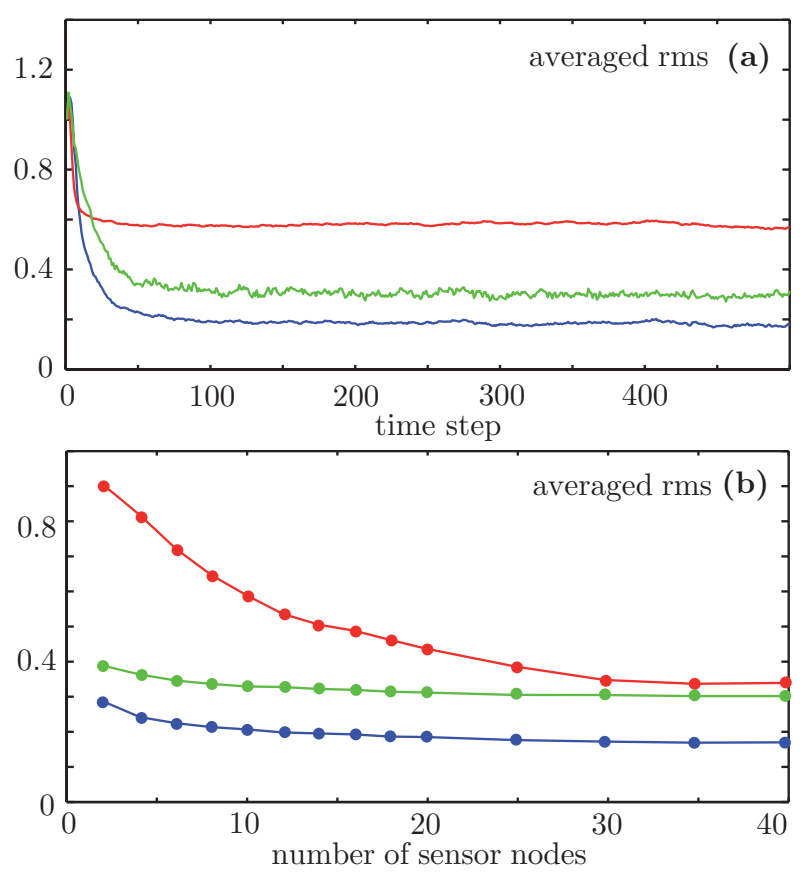

Fig. 6. Simulation results from 100 Monte Carlo simulation runs of the reconstruction process based on centralized Kalman filter (blue), decentralized Kalman filter with neglected correlations (red), and Covariance Bounds filter (green). (a) Root mean square error (rms) averaged over all discretization nodes. (b) Rms depending on the number of randomly chosen sensor nodes for each step.

S. Julier and J.K. Uhlmann. Using Covariance Intersection for SLAM. Robotics and Autonomous Systems, 55:3-20, 2007.

G. E. Karniadakis and S. Sherwin. Spectral/hp Element Methods for Computational Fluid Dynamics. Oxford University Press, 2005.

Cassio. G. Lopes and Ali H. Sayed. Incremental Adaptive Strategies Over Distributed Networks. IEEE Transactions on Signal Processing, 55:4064-4077, 2007.

Arthur G. Mutambara. Decentralized Estimation and Control for Multisensor Systems. CRC Press Inc, 1998.

M. Rabbat and R. Nowak. Distributed Optimization in Sensor Networks. In International Conference on Information Processing in Sensor Networks (IPSN 2004), 2004.

L. A. Rossi, B. Krishnamachari, and C.-C.J. Kuo. Distributed Parameter Estimation for Monitoring Diffusion Phenomena Using Physical Models. In First Annual IEEE Communications Society Conference on Sensor and Ad Hoc Communications and Networks (SECON), pages 460-469, Los Angeles, USA, 2004

Felix Sawo, Kathrin Roberts, and Uwe D. Hanebeck. Bayesian Estimation of Distributed Phenomena Using Discretized Representations of Partial Differential Equations. In Proceedings of the 3rd International Conference on Informatics in Control, Automation and Robotics (ICINCO 2006), Setubal, Portugal, 2006.

Felix Sawo, Marco F. Huber, and Uwe D. Hanebeck. Parameter Identification and Reconstruction Based on Hybrid Density Filter for Distributed Phenomena. In 10th International Conference on Information Fusion (Fusion 2007), Quebec, Canada, July 2007. 